\title{
Pressure-Induced Locking of Methylammonium Cations versus Amorphization in Hybrid Lead lodide Perovskites
}

\author{
Adrián Francisco-López,1 Bethan Charles,2 Oliver J. Weber, 2 M. Isabel Alonso, 1 Miquel \\ Garriga 1, Mariano Campoy-Quiles,1 Mark T. Weller,2 and Alejandro R. Goñi*,1,3 \\ 1Institut de Ciència de Materials de Barcelona (ICMAB-CSIC), Campus UAB, 08193 \\ Bellaterra, Spain \\ ${ }_{2}$ Department of Chemistry \& Centre for Sustainable Chemical Technologies, University \\ of Bath, Claverton Down, Bath BA2 7AY, U.K.
}

3 ICREA, Passeig Lluís Companys 23, 08010 Barcelona, Spain

\begin{abstract}
The structural phase behavior of high-quality single crystals of methylammonium lead iodide (CH3NH3PbI3 or MAPbI3) was revisited by combining Raman scattering and photoluminescence (PL) measurements under high hydrostatic pressure up to ca. $10 \mathrm{GPa}$. The single crystals were specially grown with the final thickness needed for pressure experiments, retaining their high quality due to a less invasive preparation procedure, which avoids sample thinning. Both PL and Raman spectra show simultaneous changes in their profiles that indicate the occurrence of three phase transitions subsequently at around 0.4, 2.7, and 3.3 GPa. At the second phase transition, the Raman spectra exhibit a pronounced reduction in the line width of the phonon modes of the inorganic cage, similar to the changes observed at the tetragonal-to-orthorhombic phase transition occurring at around $160 \mathrm{~K}$ but ambient pressure. This behavior is interpreted as evidence for the locking of the organic cations in the cage voids above $2.7 \mathrm{GPa}$ due to the reduced volume and symmetry of the unit cell. At the third phase transition, reported here for the first time, the PL is greatly affected, whereas the Raman spectrum experiences only subtle changes related to a splitting of some of the peaks. This behavior may indicate a change mostly in the electronic structure with little effect on the crystal structure. Strikingly, the sharp Raman features observed at high pressures do not support amorphization of MAPbI 3 with onset at $3 \mathrm{GPa}$, as claimed by most of the high-pressure (X-ray) literature. We interpret this apparent discrepancy in terms of the degree of disorder introduced at different length scales in the perovskite lattice by the pressure-induced freeze-out of the methylammonium cation motion.
\end{abstract}

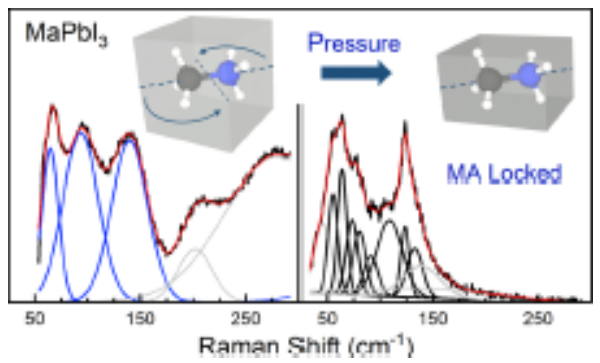




\section{INTRODUCTION}

For all of the spectacular developments in lead halide-perovskite-based photovoltaics that catapulted solar-cell efficiency to values in excess of $22 \%$ in a few years, 1 there are still several fundamental physical questions that remain a matter of debate. For example, the existence and stability of bound electron-hole pairs (excitons) and their role in the optoelectronic properties of these materials 2 remain con- troversial. Another issue is the apparent contradiction of having strong optical absorption as for direct gap materials and simultaneously the long charge-carrier lifetimes typical of indirect semiconductors like Si. A possible explanation relies on a mixed nature of the fundamental band gap in methylammonium (MA, CH3NH3) lead halide perovskites due to large spin-orbit coupling.3 Furthermore, the great advantage of this family of materials of being solution- processable is somewhat counterbalanced by their mechanical softness 4 and the consequent low energetic barri defect formation and ion migration.5,6 It is speculated that the photoinduced movement of ions and their accumulation at interfaces are the origin of large hysteresis in current-voltage characteristics 7 and transitory changes in the optical response of lead halide perovskites.8,9 Probably the most prominent drawback of these perovskite materials, however, is the sensitivity to ambient conditions, in particular humidity,10,11 which still hampers the improvement of their longtime stability. To address all of these open questions, a deeper understanding of different structure-property relationships appears necessary if not mandatory.

In this respect, high-pressure techniques offer the possibility

to vary interatomic and intermolecular distances in condensed matter in a controlled way, having large impact on the structural and consequently the electronic and optical properties of the solid. The incipient high-pressure work on hybrid halide perovskites has been recently reviewed regarding their power for assessing new properties or enlightening fundamental physical problems in this new class of photo-voltaic materials.12,13 Regarding the archetypal hybrid perov-skite MAPbI3, most of the high-pressure work performed so far concerning the structural changes under compression relied mainly, but not exclusively, on X-ray diffraction methods. Reports span different forms of the perovskite material (powder,14,15 single,14-17 or polycrystals18 and nanorods19) and different hydrostatic conditions regarding the pressure transmitting medium. The general picture that emerges for MAPbI3 is that the material is relatively soft, mainly against the tilting of the PbI6 octahedra, because it exhibits a first structural phase transition at a relatively low pressure in the range of $0.3-0.6 \mathrm{GPa}$. In addition, a second structural transformation takes place at around $3 \mathrm{GPa}$ into a very disordered phase, at which the organic cations seem to play a role. The reports, however, differ in the phenomenology at higher pressures. For the high-pressure phase, after the first transition, an orthorhombic15,17,19 as well as a cubic14,16,18 symmetry was proposed. There is more consensus about the amorphous nature of the high-pressure phase after the second phase transition. The accurate determination of the structural changes in MAPI3 of Szafranski and Katrusiak16 provides strong evidence that under hydrostatic pressure the phase changes are from tetragonal to cubic, followed by an isostructural transition to another cubic phase, which exhibits gradual amorphization with increasing pressure. Qualitatively, a similar phenomenology is found for other members of the hybrid halide perovskite family such as $\mathrm{MAPbBr} 3,14,15 \mathrm{MAPbCl} 3,20$ and $\mathrm{FAPbBr} 3,21$ where FA stands for formamidi-nium (CH(NH2)2).

As pointed out by Postorino and Malavasi,12 despite the fact that the application of high pressure is a particularly suitable means to study the interplay between degrees of freedom of the organic molecules and the inorganic cage, such kind of studies remain scarce. In recent temperaturedependent experiments, we have found in the Raman scattering signature of MAPI3 evidence of significant coupling between the vibrations of the anionic (PbI3-)n network and the $\mathrm{CH} 3 \mathrm{NH} 3+$ cations, 22,23 in spite of the supposedly weak hydrogen bonding between them. At around $160 \mathrm{~K}$, MAPbI3 undergoes a transition from the tetragonal, room-temperature phase into an orthorhombic one.24 Neutron scattering measurements provided quantitative insight into the rotational dynamics and degree of order of the MA cations within the inorganic cage in the different phases.25,26 Furthermore, time-resolved vibrational spectroscopy has identified fast librations (300 fs) and slow rotations ( $3 \mathrm{ps)}$ of the MA molecule in the tetragonal phase.27 These excitations dominate the dynamics of the MA molecules in the tetragonal phase because they are fully unlocked within the cage voids. As a consequence of the hydrogen-bonding-mediated 
interaction between the MA molecules and the halogen atoms of the inorganic cage, the vibrational modes of the latter exhibit a wide statistical distribution of frequencies, which in turn leads to a strong inhomogeneous broadening of the Raman peaks.22,23 In contrast, in the low-temperature orthorhombic phase, the reduced volume and symmetry of the cage voids causes the locking of the organic cations, which become well oriented along high-symmetry directions of the perovskite crystal. The result is a pronounced reduction of the inhomogeneous broadening, which is readily observed in low-temperature Raman spectra.22,23 Although not proven, it is believed that such a strong coupling between organic and inorganic degrees of freedom is the origin of the extremely low thermal conductivity of MAPbI3.2828 This served as motivation to look for a similar effect induced through the application of pressure rather than by decreasing the temperature, eventually leading to control of thermal transport by strain.

In this work, we present a systematic study of the structural phase behavior of high-quality $\mathrm{MAPbI} 3$ single crystals under compression by monitoring the pressure-induced changes in the fundamental band gap and vibrational spectrum, as observed in photoluminescence (PL) and Raman experiments, respectively. In the range up to ca. $10 \mathrm{GPa}$, we find three successive phase transitions characterized by sudden jumps in the energy position of the main PL emission peak, indicating abrupt changes in the fundamental band gap. A striking result is that we do not seem to observe pressure-induced amorphization of MAPbI3 in the whole pressure range of the experiment, in contrast to what might be expected from the literature. On the contrary, at the second phase transition occurring at about $2.7 \mathrm{GPa}$, the Raman spectra exhibit a clear reduction of the peak widths. As explained below, this is taken as compelling evidence of a strong reduction of phonon inhomogeneous broadening due to the pressure-induced locking of the MA cations along certain directions within the inorganic cage voids. Our ansatz is that this freeze-out of the organic cation motion leads locally to an orthorhombic distortion of the crystal structure of MAPbI3, as probed by Raman scattering, whereas on a macroscopic scale, the different locked orientations and positions of the MA molecules disrupt the long-range order sensed by X-rays. The latter has been repeatedly interpreted as amorphization. Moreover, at the third phase transition, which takes place at ca. 3.3 GPa, only the emission properties are strongly affected, whereas the Raman spectra barely display a slight splitting of a couple of modes. We believe that this phenomenon is indicative of subtle structural changes at the phase transition, which despite their small magnitude can cause a partial folding of the Brillouin zone (BZ) in the high-pressure phase. As a consequence, the gap widens suddenly from 1.85 to $2.15 \mathrm{eV}$.

\section{EXPERIMENTAL SECTION}

\section{Preparation of Loading-Ready Hybrid Perovskite}

Single Crystals. The methylammonium iodide (MAI) synthesis proceeds by adding dropwise $16.620 \mathrm{~mL}$ of $\mathrm{HI}(57 \mathrm{wt} \% \mathrm{aq}+1.5 \%$ H3PO2 stabilizer) to $10.891 \mathrm{~mL}$ of methylamine (40 wt \% aq) in $50 \mathrm{~mL}$ of isopropanol in an ice bath under stirring. The solvent is removed by rotary evaporation, and the crude product recrystallizes from hot ethanol, which is then washed with diethyl ether and dried at $100{ }^{\circ} \mathrm{C}$. The space-confined on-substrate fabrication method of MAPbI3 single crystals with adjustable thickness 29 consists first in dissolving $2.3050 \mathrm{~g}$ of PbI2 (99\%, Sigma) and $0.7948 \mathrm{~g}$ of MAI in $5 \mathrm{~mL}$ of dry $\gamma$-butyrolactone in a normal fume cupboard, affording a 1 $\mathrm{mol} / \mathrm{dm} 3$ solution. Then, $100 \mu \mathrm{L}$ of the solution is dropped onto a glass coverslip on a hotplate. Another glass coverslip is placed on top of the first, sandwiching the solution between. The hotplate is ramped to $100{ }^{\circ} \mathrm{Cat} 10{ }^{\circ} \mathrm{C} / \mathrm{h}$ and retained at $100{ }^{\circ} \mathrm{C}$ for $48 \mathrm{~h}$, driving off the solvent and inducing crystallization of flat MAPbI3 crystals. The thickness of the crystals was varied by application of pressure using a Petri dish of weight $120 \mathrm{~g}$ applied to substrates. The dimensions of the crystals were found to be $2.0 \mathrm{~mm}$ (width) by $32 \mu \mathrm{m}$ (depth) through parallax measurements taken using secondary electron images from a scanning electron microscope.

High-Pressure Experiments. The high-pressure photo-luminescence and micro-Raman scattering measurements were performed at room temperature employing a gasketed diamond anvil cell (DAC). Anhydrous propanol was used as pressure transmitting medium, which ensures good hydrostatic conditions (perfectly hydrostatic up to $4.2 \mathrm{GPa} 30$ ) and proved to be chemically inert to 
MAPbI3. Thanks to the growth procedure that directly produced single crystals with a thickness of ca. $30 \mu \mathrm{m}$ and there was no need to thin down the sample for loading the DAC. This is very important because the thinning procedure employing either mechanical polishing or chemical etching is known to spoil the quality of such soft crystals like MAPbI3, introducing large amounts of structural defects, which might have an influence on the behavior of the material under compression. Small pieces cut with a sharp knife of about $100 \times 100 \mu \mathrm{m} 2$ in size were placed into the DAC together with a ruby sphere for pressure calibration.31 The PL spectra were excited with the $633 \mathrm{~nm}$ line of a He-Ne laser and the $514.5 \mathrm{~nm}$ line of an Ar+-ion laser at pressures lower and higher than $3.5 \mathrm{GPa}$, respectively, whereas the infrared diode laser emitting at $785 \mathrm{~nm}$ was employed for the Raman measurements. The latter turned out most suitable to excite the vibrational modes of the inorganic cage, providing also the highest spectral resolution and straylight rejection. In all cases, a very low incident light power density below $15 \mathrm{~W} / \mathrm{cm} 2$ was used to avoid any photo-degradation of the samples, such that thermal damage by the laser can be safely ruled out. Spectra were collected using a $20 \times$ long working distance objective with NA $=0.35$ and dispersed with a high-resolution LabRam HR800 grating spectrometer equipped with a chargecoupled device detector. PL spectra were corrected for the spectral response of the spectrometer by normalizing each spectrum using the detector and the 600 grooves $/ \mathrm{mm}$ grating characteristics.

\section{RESULTS AND DISCUSSION}

\section{Photoluminescence Measurements under Pressure.}

Figure 1 shows the evolution of the PL spectra of MAPbI3 with increasing pressure. All spectra were normalized to their absolute maximum intensity and vertically offset to ease their comparison. It is worth mentioning that we loaded two pieces of MAPbI 3 inside thediamond anvilcell(DAC) and constituently performed the PL and Raman measurements at the same two points on each piece. The results of the four measurement series are similar; hence, we only show the representative ones. The main PL peak exhibits sudden jumps in the position of its maximum, which are indicative of the occurrence of three phase transitions in the pressure range of the experiment. Different colors correspond to the four observed phases, and the same code will be used throughout the article. At the beginning of the pressure upstroke and before the first phase transition occurs, the PL spectra (blue full lines) exhibit a slight but clear red shift, which indicates that the fundamental band gap of MAPbI3 in the room-temperature tetragonal phase decreases with pressure. The first phase transition starts at a very low pressure of $0.4 \mathrm{GPa}$, as signaled by an abrupt blue shift of the main PL peak. This transition is completed at about $0.7 \mathrm{GPa}$, before which both

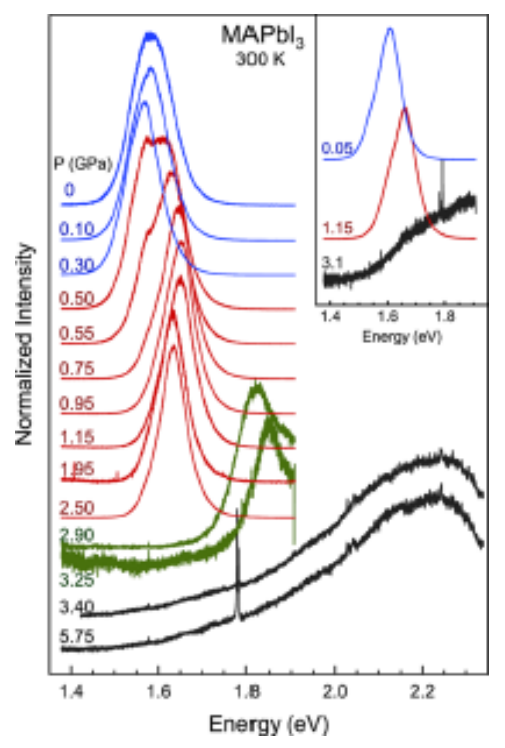


Figure 1. PL spectra of MAPbI3 obtained for incremental steps of pressure. The spectra were normalized to their maximum intensity and plotted with a vertical shift for increasing pressure. The different colors indicate the subsequent phases adopted by the material during the pressure upstroke. For pressures lower and higher than $3.4 \mathrm{GPa}$, the excitation laser wavelengths were 633 and $514 \mathrm{~nm}$, respectively. The inset displays spectra measured by releasing the pressure, demonstrating the full reversibility of the structural changes except for some hysteresis.

phases coexist. The new high-pressure phase is stable up to $2.7 \mathrm{GPa}$ (red solid curves) and should correspond to the cubic phase IV, as reported elsewhere.16 With increasing pressure and within this phase, the PL emission exhibits first a gradual blue shift and finally a red shift. At about 2.7 $\mathrm{GPa}$, a second phase transformation occurs, which is characterized by an abrupt but stronger blue shift of the PL maximum (green solid curves). As explained in the discussion of the Raman results, this new phase is perfectly crystalline and probably orthorhombic in nature. This result is at odds with the widespread claims of this being the amorphous cubic phase

V.12,16 The PL spectra clearly indicate the occurrence of a third phase transition at about $3.3 \mathrm{GPa}$, which is again characterized by an abrupt blue shift and a pronounced broadening of the emission peak (black spectra in Figure 1). To properly excite the PL of this phase, we switched to the green laser line. We anticipate that the Raman data indicate again that this phase is crystalline and probably orthorhombic up to the highest pressure of this experiment close to $6 \mathrm{GPa}$. The broadband emission exhibited by this high-pressure phase, though, speaks for a large heterogeneity in the sample, as far as the electronic states involved in the optical transitions are concerned. It is important to remark that all changes in the PL emission (the Raman too) are fully reversible, even though there is a certain degree of hysteresis, as illustrated by the PL spectra of the inset to Figure 1 , which were taken by releasing the pressure prior to the final opening of the DAC (see also Figure S2 of the Supporting Information).

To analyze the PL spectra of MAPbI3, we used a Gaussian- Lorentzian cross-product function for describing the main peaks, which are ascribed to free-exciton recombination. We notice that the same fitting protocol was also successfully employed for the analysis of the PL spectra of MAPbI3 at ambient pressure but as a function of temperature (data will be published elsewhere32). The expression for the cross-product function reads as

$$
\begin{aligned}
G \times L(\hbar \omega)= & A \cdot \frac{\Gamma^{2}}{4 \cdot s \cdot\left(E_{0}-\hbar \omega\right)^{2}+\Gamma^{2}} . \\
& \exp \left(-4 \ln 2 \frac{(1-s) \cdot\left(E_{0}-\hbar \omega\right)^{2}}{\Gamma^{2}}\right)
\end{aligned}
$$

where $\mathrm{A}$ is the amplitude prefactor, $\mathrm{E} 0$ is the peak energy position, $\Gamma$ is the full width at halfmaximum (FWHM), and $\mathrm{s}$ is a weight parameter that takes the value $\mathrm{s}=0$ for pure Gaussian lineshape and $\mathrm{s}=1$ for pure Lorentzian. This function is a useful simplification of a Voigt function, which corresponds to the mathematical convolution of a Lorentzian, taking into account the natural lineshape due to spontaneous emission and whose line width is the inverse of the lifetime, and an inhomogeneously broadened Gaussian, accounting for a normal distribution of exciton energies. Apart from yielding an excellent lineshape fit, the cross-product function has the advantageagainst theVoigtfunctionofbeingfarless computationally expensive. Representative examples of the lineshape analysis of the PL profiles are depicted in Figure S1 of the Supporting Information.

The assignment of the main emission peak to free-exciton recombination is partly controversial because the reported values for the exciton binding energy in lead halide perovskites span a wide range from a few millielectronvolts $33-40$ to several tens of millielectronvolts.41-44 As suggested,2 such a difficulty to discern between the free-carrier or correlated electron-hole pair character of the optical properties in these materials might be in direct relation to the particular microstructure of the sample being considered. The reason is that the dielectric screening and consequently the magnitude of the exciton binding energy are largely affected by the presence of grain boundaries, 
interfaces, and surface defects, where charge can accumulate or dipoles can be induced, all impacting the macroscopic polarization of the medium.2,34 Furthermore, we point out that claims for the absence of exciton states based on a steplike absorption edge with no peaklike feature at its edge are partly incorrect.2,33,38,45 A steplike profile is the evidence of Coulomb-interactionmediated correlations between electrons and holes in the states pertaining to the excitonic energy continuum.33,46 The absence of a peak just means the absence of bound excitonic states corresponding to the discrete energy spectrum, most probably because the exciton binding energy is on the order of or smaller than the thermal energy. This, in contrast, does not hinder bound exciton states in playing a main role in emission because their optical recombination is favored against that of continuum states due to the much shorter lifetime of the latter.47 We thus use the Gaussian- Lorentzian cross-product function of eq 1 to describe the lineshape of the PL emission, which we ascribe to free-exciton recombination.

The values of the fitting parameters corresponding to the energy E0 of the peak maximum and the integrated intensity, given by the product $A \cdot \Gamma$, are plotted as a function of pressure in Figure 2. Again, the abrupt changes in the parameters clearly

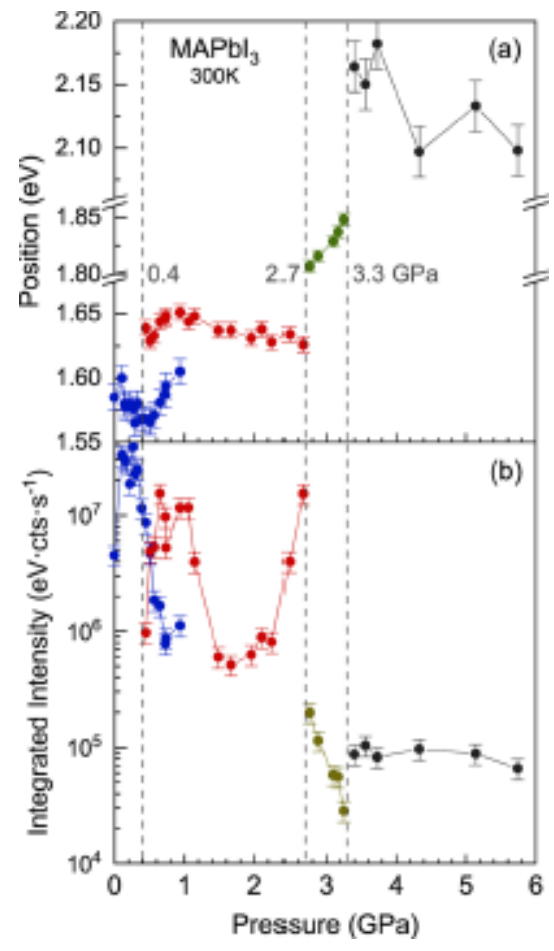

Figure 2. (a) Maximum peak position and (b) integrated intensity plotted as a function of pressure, obtained from the PL lineshape fits using eq 1. The pressures at which the phase transitions occur are indicated by vertical dashed lines.

indicate the pressures at which the phase transitions occur (marked by dashed vertical lines). 48 Although we cannot tell the absolute values of band gap and/or exciton binding energy from the lineshape fits, the shift of the PL peak energy E0 with pressure is to a large extent dictated by the pressure dependence of the gap. In particular, we analyze the pressure-induced red shift of the gap in the tetragonal phase of $\mathrm{MAPbI} 3$, stable at ambient conditions, which is apparently at odds with the well-established systematics on the pressure dependence of direct band gaps in tetrahedrally bonded semiconductors.46 In brief, for semiconductors with predom-inantly sp3 hybridization, it holds that (i) states with bonding

p-orbital character are almost insensitive to pressure. The best example is the top of the valence band at the $\Gamma$ point of the Brillouin zone (BZ), which is bonding pure p-type. (ii) On the contrary, antibonding s-states exhibit a strong blue shift with pressure. In most of the cases, the bottom of the conduction band also at $\Gamma$ comprises states with antibonding pure s-orbital character. In consequence, direct gaps at the BZ center increase with pressure at a rate on the order of 100 
meV/ GPa.46 (iii) Antibonding p-orbitals are characterized by a much smaller but negative deformation potential as compared with s-states. Antibonding p-like states contribute the most to the $\mathrm{X}$-valleys at the $\mathrm{BZ}$ edge. As a result, the indirect $\Gamma-\mathrm{X}$ gap decreases with pressure at a lower pace of typically $-15 \mathrm{meV} / \mathrm{GPa} .46$ Relativistic band-structure calculations 35,49,50 for a pseudocubic phase of MAPbI3 predict a direct fundamental gap at the R-point of the BZ. The peculiarity of lead halide perovskites is that due to the huge spin-orbit interaction present in heavy atoms like $\mathrm{Pb}$ and $\mathrm{I}$ there is a so-called band inversion such that the top of the valence band is predominantly composed by antibonding $\mathrm{Pb}$ 6s orbitals hybridized with I 5p orbitals and the bottom of the conduction band is formed by the antibonding split-off $\mathrm{Pb} 6 \mathrm{p}$-orbitals. Assuming that a similar atomic orbital behavior to that of conventional semiconductors holds for these perovskites, one would expect that the top of the valence band shifts up and the bottom of the conduction band shifts down slightly with applied pressure, leading to a reduction of the fundamental gap, as experimentally observed (linear pressure coefficient of $-60(10) \mathrm{meV} / \mathrm{GPa}$ ).

Raman Measurements under Pressure. Figure 3 summarizes the Raman results obtained on singlecrystalline

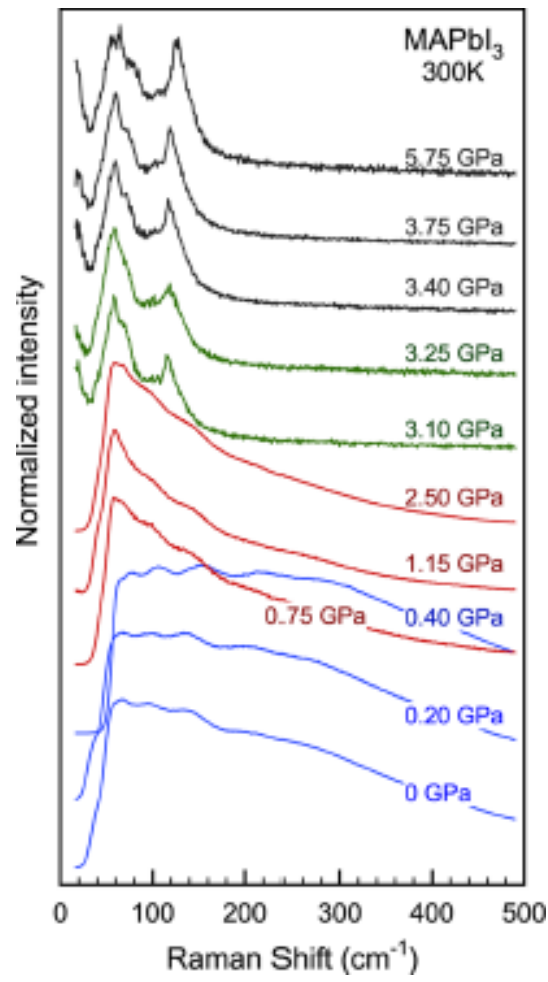

Figure 3. Room temperature Raman spectra of MAPbI3 measured at different pressures. The spectra were normalized to their maximum intensity and shifted vertically for clarity. The different colors of the spectra indicate the changes in phase after every transition.

$\mathrm{MAPbI} 3$ as a function of hydrostatic pressure. Once more, to ease the comparison, the spectra were normalized to their maximum intensity and plotted with a vertical offset with increasing pressure. The colors of the curves correspond to the different phases, as determined from the PL measurements, because the occurrence of the pressure-induced structural transitions cannot always be clearly established from the occasionally subtle changes in the Raman spectra. For a quantitative assessment of the effect of pressure on the vibrational spectrum of MAPbI3 we have decomposed it in its different mode components by a lineshape analysis, as illustrated in Figure 4, with a representative example of the 


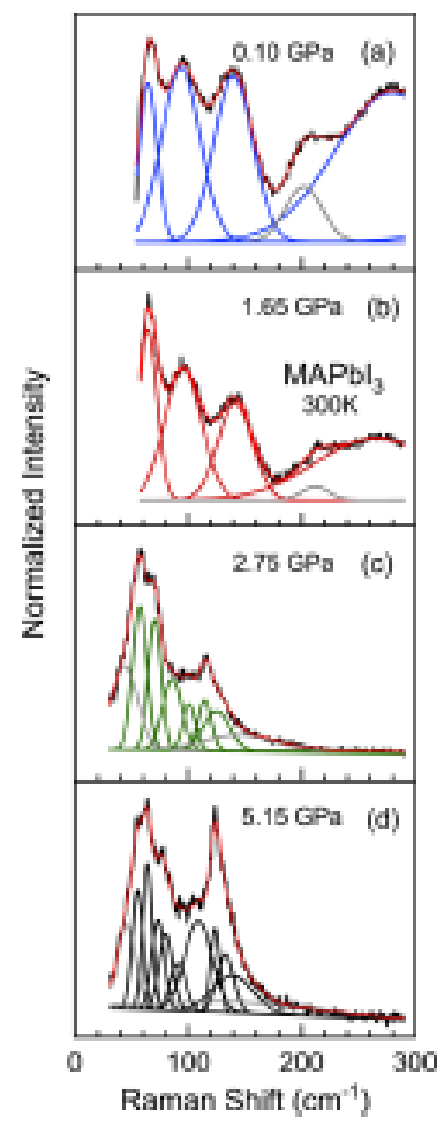

Figure 4. Examples of the performed lineshape fits to the Raman spectra using Gaussian functions for different pressures: (a) $0.10 \mathrm{GPa}$,(b) $1.65 \mathrm{GPa}$, (c) $2.75 \mathrm{GPa}$, and (d) $5.15 \mathrm{GPa}$, each one representing adifferent high-pressure phase of the material. We point out that the number of Gaussians used for the fits is compatible with the Raman active modes predicted by theory (out of 18 cage eigenmodes) 22 and those observed at low temperature for the orthorhombic phase of MAPbI.23 The gray solid curves are auxiliary peaks used to improve the fits and are not discussed further. Spectra shown in panels (a) and (b) were obtained by subtracting the broad PL peak from the Raman raw data (see the text for details).

fits for each of the observed phases. We note that for the first two phases $(\mathrm{P}<2.7 \mathrm{GPa})$ all Raman peaks appear on top of a very broad but intense peak, which is ascribed to the main excitonic emission (Figure 3). In fact, the qualitative changes in the Raman spectra of the first two phases are principally due to the shift of this broad background, which varies under compression in exactly the same way as the main excitonic PL peak. For completeness, we refer to a possible alternative explanation in which this broadband is interpreted as a broad central Raman peak originating from local polar fluctuations intrinsic to the perovskite structure.51 The strong PL background, however, does not hamper the observation of the Raman features because the latter become resonantly enhanced (outgoing resonance), when the $785 \mathrm{~nm}$ line is used for excitation. As for the PL analysis, the background was described by a Gaussian-Lorentzian cross-product function, which has been subtracted from the Raman spectra of the two lowest-pressure phases (see Figure 4a,b) for a better visualization of the phonon modes. All Raman bands/peaks have been fitted using Gaussian functions. On the basis of a previous full assignment of Raman22,23 and far-infrared spectra,52 all Raman peaks with frequency lower than $200 \mathrm{~cm}-1$ correspond to optical phonons of the inorganic cage, whereas the broad peak centered at around $280 \mathrm{~cm}-1$ is assigned to the so-called isolated mode associated with a torsional deformation of the MA molecules.

The results of the Raman lineshape fits for the mode frequencies and full widths at half-maximum are depicted in Figure 5 as a function of pressure. The first transition from the 


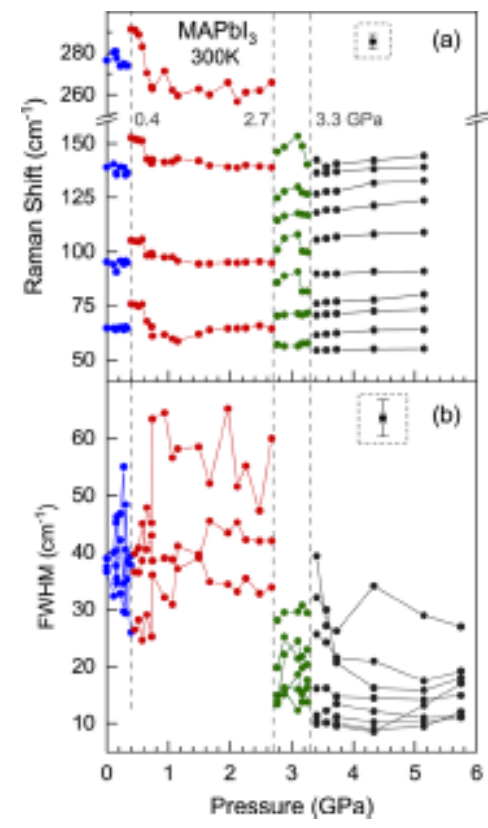

Figure 5. (a) Frequency and (b) full width at half-maximum (FWHM) of the Raman peaks, as obtained from the lineshape fits, as a function of pressure up to ca. $6 \mathrm{GPa}$. The pressures at which the phase transitions occur are marked with vertical dashed lines. Representative error bars are indicated by the symbols inside the dashed squares.

tetragonal to cubic phase16 can be inferred from the subtle but abrupt changes in the mode frequencies observed at $0.4 \mathrm{GPa}$, coinciding with the pressure at which the different PL contributions start to change. In contrast, the occurrence of the second phase transition at $2.7 \mathrm{GPa}$ is clearly revealed by the abrupt reduction of the width of all Raman peaks. It is very difficult to discern if each of the broad bands observed in the Raman spectra of the cubic high-pressure phase splits into several sharp peaks, indicating a reduction of crystal symmetry, or the different components are simply spectrally resolved above $2.7 \mathrm{GPa}$ due to the much smaller widths. Hence, we cannot tell with any degree of confidence if the third phase is cubic, tetragonal, or orthorhombic. Finally, at $3.3 \mathrm{GPa}$, a third phase transition takes place. An abrupt blue shift of the main PL band occurs, whereas in Raman spectra, the transition can only be assessed by closer inspection of the fitting results (see Figure 5). Above $3.3 \mathrm{GPa}$, the Raman peaks sharpen further but gradually and some of the peaks split into two. Thus, additional Gaussians ought to be included in the fitting function to describe the Raman lineshape accurately. Such a contrasting behavior of the radiative recombination and the vibrational properties of MAPbI3 under high pressure speaks for a transition to a phase that exhibits a slight lattice distortion concomitant with a strong alteration of the electronic properties. Are we being confronted with a kind of "dimerization" of the lattice or the appearance of a super-structure of the organic cations, similarly to the pressure-induced, magnetically driven Peierls transition in $\mathrm{CuGeO} 3,53$ to give just one example? Clearly more experimental as well as theoretical work is needed to answer this question.

We now turn to the discussion of one of the main results of this work, which concerns the pronounced sharpening of all Raman features taking place at the second phase transition at 2.7 GPa. Such a behavior of the line widths is totally similar to the one observed for decreasing temperature at the tetragonal-to-orthorhombic transition.22,23 On the basis of this similarity, we propose that the line width reduction of the Raman peaks has the same origin as for the lowtemperature orthorhombic phase of MAPbI3. The reduced symmetry of the structure in the highpressure phase together with a smaller void volume leads to the locking of the MA molecules inside the inorganic cage voids in certain crystalline directions. This causes a sudden and marked reduction of the inhomogeneous broad-ening of the Raman modes, as a consequence of the vanishing dynamical disorder of the organic cations when locked. This is an important result implying that the suppression of dynamical disorder, which is entropically favorable at low temperatures, is also achieved at room temperature through pure mechanical deformation of the single crystal. 
Irrespective of its origin, the reduction of the dynamical disorder should have the same positive impact on the thermal conductivity of the material.28 On the contrary, in an amorphous or highly disordered phase, the thermal conductivity would remain constant or even decrease.

At this point, we would like to call attention to a striking result, namely, the fact that, in our case, $\mathrm{MAPbI} 3$ does not seem to transform isostructurally into another cubic but amorphous phase at around 3.0 GPa.16 The sharp peaks we observe in Raman spectra up to $10 \mathrm{GPa}$, the highest pressure in our experiment, clearly speak against amorphization, defying the claims of the literature.14-19 Although we cannot tell the symmetry of the lattice by inspection of just the Raman data, as explained below, we found an unexpectedly strong parallel with the structural behavior of $\mathrm{PbI} 2$ under pressure. We truly believe that the different behaviors observed for $\mathrm{MAPbI} 3$ under pressure strongly depend on the crystal quality of the sample loaded into the DAC and, of course, of the hydrostatic conditions provided by the particular pressure transmitting medium being used, if any. Regarding the former issue, we emphasize that the single crystals used here are unique because they were specifically grown with the desired final thickness to fit into the DAC. In this sense, except for the mild cutting into smaller pieces, our crystals have not suffered any mistreatment concerning the necessary thinning of the samples to be loaded into the DAC. It is likely that mechanical polishing or chemical etching might introduce structural defects in a soft material like MAPbI3, which under compression would stabilize other phases, leading to a different structural path as a function of pressure.

There is, however, another possibility to explain the apparent discrepancy between our highpressure Raman results and the X-ray diffraction data of the literature. The crystallographic information extracted from the diffraction patterns depends much on having high long-range order in the structure. On the contrary, Raman scattering is much less sensitive to disorder because even vibrations with an extremely short coherence length are detected. Good examples are the sharp features observed in Raman spectra of semiconductor alloys54 or the molecular vibrations of the MA cations, 23 for instance. Hence, it could be that the high-pressure phase of MAPbI 3 above 3 GPa corresponds to one in which the pre-existing dynamical disorder of the MA cations is "frozen" at the phase transition. This results in a kind of polycrystal regarding the strain inhomogeneity with nanometric-size domains with different deformation directions such that the long-range order of the inorganic lattice is sufficiently disturbed to affect the $\mathrm{X}$-ray patterns but not the Raman features.

A few words about the pressure-induced structural effects on MAPbI3 are in place here. We have run further pressure experiments up to $10 \mathrm{GPa}$ and observed that the degree of structural disorder, as indicated by the magnitude of the line broadening of the Raman peaks of the phase setting at above $2.7 \mathrm{GPa}$, which is sometimes taken for amorphization, depends on how fast pressure is increased. When approaching the phase transition pressure, a rapid increase of pressure leads to broader Raman features, indicating a more disordered structure. If the sample is left overnight at a pressure slightly higher than $2.7 \mathrm{GPa}$, the Raman spectra measured the next day are as sharp as the ones shown in Figure 3. This clearly indicates that the structural disorder in MAPbI3, as well as the occurrence of the fourth phase transition at ca. $3.3 \mathrm{GPa}$, is a kinetically limited process.

At this point, we would like to report on an accidental finding that might have implications for the understanding of the high-pressure structural behavior of MAPbI3. In another experiment, we loaded the DAC using the usual 4:1 methanol/ethanol mixture. Most likely due to the presence of water traces in the mixture, $55 \mathrm{MAPbI} 3$ unequivocally transformed into $\mathrm{PbI} 2$ under compression at a moderate pressure of about $1 \mathrm{GPa}$. In Figures S3 and S4 of the Supporting Information, we display all of the Raman spectra as taken in a chronological order and compare them with Raman results for $2 \mathrm{H}-\mathrm{PbI} 2$ under pressure.56 The similarity of the Raman spectra is so striking that leaves no doubts that the crystal had already transformed into $\mathrm{PbI} 2$ when pressure was released to almost ambient after the first upstroke. Moreover, during the second upstroke and at around $0.6 \mathrm{GPa}$, the phase transition from the two-dimensional (layered) form of $2 \mathrm{H}-\mathrm{PbI} 2$ into a three-dimensional crystal structure is clearly observed, exactly as reported.56 Above $5.5 \mathrm{GPa}$, however, we find another phase transformation of $\mathrm{PbI} 2$, not previously reported. As illustrated in Figure 6, the Raman spectra of $\mathrm{PbI} 2$ at such high pressures 


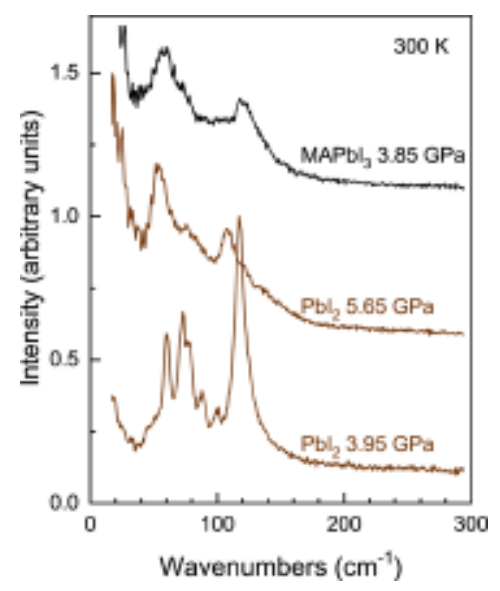

Figure 6. Raman spectrum of MAPbI3 at $3.85 \mathrm{GPa}$ compared to the spectra of PbI2 (transformed from $\mathrm{MAPbI} 3$ under pressure) at 3.95 and $5.65 \mathrm{GPa}$, corresponding to two different high-pressure phases of the latter. The resemblance of the Raman spectra of the highest-pressure phases of MAPbI3 and the one of PbI2 is astonishing.

and those of MAPbI3 in the phase where the MA molecules are locked in the cage voids are astonishingly similar. Although both materials are certainly different (see Supporting Information for details), we are lead to the conclusion that they must share similar structural units in these highpressure phases. On the one hand, the full reversibility of the structural changes in MAPbI3 provides evidence that the sixfold coordination of the $\mathrm{Pb}$ atoms to the iodides is preserved and the high-pressure phases differ from each other only in the degree of tilting and deformation of the corner-shared PbI6 octahedra. Furthermore, due to the observed locking of the MA molecules in the voids, the phase setting at above $2.7 \mathrm{GPa}$ is likely to be orthorhombic. On the other hand, recent searching simulations performed through the swarm-intelli-gence CALYPSO method57 predict for $2 \mathrm{H}-\mathrm{PbI} 2$ a series of phase transformations from the layered $\mathrm{P} \overline{\mathrm{m}} 1$ structure to an orthorhombic Pnma and a tetragonal I4/mmm one at 2.5 and 27 GPa, respectively. Precisely, the tetragonal phase, which is characterized by a 10 -fold coordination of PbI10 dodecahedral units, is a good candidate for the high-pressure phase we found above $5.5 \mathrm{GPa}$ in PbI2, in spite of the large discrepancy in the calculated and measured transition pressures. Hence, the similar shape of the Raman spectra of both materials displayed in Figure 6 might be due to the similarity of the structural building blocks, whereas the red shift of the mode frequencies (even for a much higher pressure) of $\mathrm{PbI} 2$ compared to that of MAPbI3 is a consequence of the lengthening of the $\mathrm{Pb}-\mathrm{I}$ bonds imposed by a higher coordination of the structure of the former material.

Finally, we would like to comment on results of a structural search of the high-pressure phases of MAPbI3, performed with the minima-hopping method and a classical force-field model, which appeared very recently in the literature. 58 The search revealed that at least two structures are denser and lower in energy than the experimentally observed low-temperature orthorhombic phase. The calculations further show that particularly a phase type called double-delta could be thermodynamically stabilized under compression in the pressure range of 2-3 GPa, i.e., the range where we observe the second and third phase transitions in MAPbI3 (at room temperature, though). Double-delta phases contain a pillarlike structure of edge-sharing PbI6 octahedra, whereas the MA cations uniformly surround them.58 Interestingly, these novel phases exhibit a much wider fundamental band gap (larger than $3 \mathrm{eV}$ ), similarly to the case of the high-pressure phase we found setting at above $3.3 \mathrm{GPa}$. From the many issues that remain unclear, the understanding of the outspoken reversi-bility of the pressure-induced structural changes seems crucial. For example, how reversible would be the transformation from the tetragonal or cubic structures, characterized by corner-shared octahedra, to phases like the double-delta ones? The formation of the pillars of edgeshared octahedra in the latter would necessarily imply massive atom movement and bond breaking, which would render difficult the reverse trans-formation. Clearly, more experimental as well as theoretical work is necessary to finally pinpoint the pressure-temperature phase diagram of $\mathrm{MAPbI} 3$. 


\section{CONCLUSIONS}

In summary, by combining optical spectroscopy (Raman and photoluminescence) with highpressure techniques, we were able to study structure-property relationships of the archetypal hybrid lead halide perovskite MAPbI3. The high-quality single crystals, explicitly grown by the space-confined method with the desired thickness for high-pressure experi-ments, exhibit a peculiar structural behavior under compres-sion characterized by a sequence of three structural phase transitions at $0.4,2.7$, and $3.3 \mathrm{GPa}$. All phases are perfectly crystalline, with more or less dynamical disorder of the MA cations, as evidenced by the Raman data. In addition, two issues of the highpressure phenomenology are worth to be emphasized: On the one hand, at the second phase transition around $2.7 \mathrm{GPa}, \mathrm{MAPbI} 3$ undergoes a transformation into a phase that is characterized by a pronounced reduction of the inhomogeneous broadening of the Raman lines. This is interpreted as evidence of a significant decrease in lattice dynamical disorder because of the locking of the organic cations inside the inorganic cage voids.23 This behavior is at odds with the previously reported smearing of X-ray diffraction patterns above $3 \mathrm{GPa}$, interpreted as amorphization. This is, however, an apparent contradiction. Concomitant with the pressureinduced locking of the MA cations, there is a local, orthorhombic distortion of the inorganic cage structure, which on a macroscopic length scale occurs randomly in different directions. At last, this inhomogeneity disturbs the long-range order of the crystal structure, leading to the smearing of the $\mathrm{X}$-ray patterns. Further investigation revealed that the degree of pressure-induced disorder not only depends on crystal quality but also is a kinetically limited process, which is prevented when pressure is slowly raised in the pressure range close to the second phase transition but favored by fast compression (see the discussion in the Supporting Information). On the other hand, the third phase transition at $3.3 \mathrm{GPa}$ involves only a slight structural change, apparent in the Raman spectra only as an additional but small decrease in the line widths and splitting of some peaks, which in turn has associated a marked widening of the fundamental gap. Consequently, the main emission peaks at a much higher energy of $2.15 \mathrm{eV}(577 \mathrm{~nm})$ and broadens significantly to a FWHM of ca. 450 $\mathrm{meV}$. The latter points to large inhomogeneities in the electronic and optical properties of this highpressure phase of MAPbI3. Our findings provide further fundamental insight into the under-lying physics of this kind of organic-inorganic hybrid materials, which are causing a change in paradigm in photovoltaic and light-emitting device technology and, possibly, for their by far less explored application in thermoelectricity.

\section{AUTHOR INFORMATION}

\section{Corresponding Author}

*E-mail: goni@icmab.es.

ORCID

Mark T. Weller: 0000-0002-7475-0379

Alejandro R. Goni: 0000-0002-1193-3063

Notes

The authors declare no competing financial interest.

\section{ACKNOWLEDGMENTS}

The Spanish Ministerio de Economía, Industria y Compet-itividad, is gratefully acknowledged for its support through Grant No. SEV-2015-0496 in the framework of the Spanish Severo Ochoa Centre of Excellence program and through Grants MAT2015-70850-P (HIBRI2) and CSD201000044 (Consolider NANOTHERM). A.F.-L. acknowledges a FPI fellowship from the Spanish Ministerio co-financed by the European Social Fund. B.C. and O.J.W. thank the EPSRC for Ph.D. studentship funding via the CSCT CDT (EP/G03768X/1, EP/L016354/1). Financial support is also acknowledged from the European Research Council through project ERC CoG648901. 


\section{REFERENCES}

(1) Yang, W. S.; Park, B.-W.; Jung, E. H.; Jeon, N. J.; Kim, Y. C.; Lee,

D. U.; Shin, S. S.; Seo, J.; Kim, E. K.; Noh, J. H.; et al. Iodide management in formamidinium-leadhalide-based perovskite layers for efficient solar cells. Science 2017, 356, 1376-1379.

(2) Grancini, G.; Kandada, A. R. S.; Frost, J. M.; Barker, A. J.; De Bastiani, M.; Gandini, M.; Marras, S.; Lanzani, G.; Walsh, A.; Petrozza, A. Role of microstructure in the electron-hole interaction of hybrid lead halide perovskites. Nat. Photonics 2015, 9, 695-700.

(3) Wang, T.; Daiber, B.; Frost, J. M.; Mann, S. A.; Garnett, E. C.; Walsh, A.; Ehrler, B. Indirect to direct bandgap transition in methylammonium lead halide perovskite. Energy Environ. Sci. 2017, 10, 509-515.

(4) Faghihnasiri, M.; Izadifard, M.; Ghazi, M. E. DFT study of mechanical properties and stability of cubic methylammonium lead halide perovskites (CH3NH3PbX3, X = I, Br, Cl). J. Phys. Chem. C 2017, 121, 27059-27070.

(5) Domanski, K.; Roose, B.; Matsui, T.; Saliba, M.; Turren-Cruz, S.-H.; Correa-Baena, J.-P.; Carmona, C. R.; Richardson, G.; Foster, J. M.; De Angelis, F.; et al. Migration of cations induces reversible performance losses over day/night cycling in perovskite solar cells. Energy Environ. Sci. 2017, 10, 604-613.

(6) Liu, J.; Yin, X.; Liu, X.; Que, M.; Que, W. Multi-influences of ionic migration on illumination-dependent electrical performances of inverted perovskite solar cells. J. Phys. Chem. C 2017, 121, 16051- 16057.

(7) Li, C.; Guerrero, A.; Zhong, Y.; Huettner, S. Origins and mechanisms of hysteresis in organometal halide perovskites. J. Phys.: Condens. Matter 2017, 29, No. 193001, and references therein.

(8) Gottesman, R.; Gouda, L.; Kalanoor, B. S.; Haltzi, E.; Tirosh, S.; Rosh-Hodesh, E.; Tischler, Y.; Zaban, A.; et al. Photoinduced reversible structural transformations in free standing CH3NH3PbI3 films. J. Phys. Chem. Lett. 2015, 6, 2332-2338.

(9) Lang, F.; Shargaieva, O.; Brus, V. V.; Neitzert, H. C.; Rappich, J.; Nickel, N. H. Influence of radiation on the properties and the stability of hybrid perovskites. Adv. Mater. 2018, No. 1702905.

(10) Leguy, A. M. A.; Hu, Y.; Campoy-Quiles, M.; Alonso, M. I.; Weber, O. J.; Azarhoosh, P.; van Schilfgaarde, M.; Weller, M. T.; Bein, T.; Nelson, J.; et al. Reversible hydration of CH3NH3PbX3 in films, single crystals, and solar cells. Chem. Mater. 2015, 27, 3397-3407.

(11) Wang, Q.; Chen, B.; Liu, Y.; Deng, Y.; Bai, Y.; Dong, Q.; Huang, J. Scaling behavior of moisture-induced grain degradation in polycrystalline hybrid perovskite thin films. Energy Environ. Sci. 2017, 10, 516-522.

(12) Postorino, P.; Malavasi, L. Pressure-induced effects in organic-inorganic hybrid perovskites. J. Phys. Chem. Lett. 2017, 8, 2613-2622.

(13) Jaffe, A.; Lin, Y.; Karunadasa, H. I. Halide perovskites under pressure: Accessing new properties through lattice compression. ACS Energy Lett. 2017, 2, 1549-1555.

(14) Jaffe, A.; Lin, Y.; Beavers, C. M.; Voss, J.; Mao, W. L.; Karunadasa, H. I. High-pressure single-crystal structures of $3 \mathrm{D}$ lead-halide hybrid perovskites and pressure effects on their electronic and optical properties. ACS Cent. Sci. 2016, 2, 201-209.

(15) Kong, L.; Liu, G.; Gong, J.; Hu, Q.; Schaller, R. D.; Dera, P.; Zhang, D.; Liu, Z.; Yang, W.; Tang, Y.; et al. Simultaneous band-gap narrowing and carrier-lifetime prolongation of organicinorganic trihalide perovskites. Proc. Natl. Acad. Sci. U.S.A. 2016, 113, 8910- 8915.

(16) Szafranski, M.; Katrusiak, A. Mechanism of pressure-induced phase transitions, amorphization, and absorption-edge shift in photovoltaic methylammonium lead iodide. J. Phys. Chem. Lett. 2016, 7, 3458-3466.

(17) Capitani, F.; Marini, C.; Caramazza, S.; Postorino, P.; Garbarino, G.; Hanfland, M.; Pisanu, A.; Quadrelli, P.; Malavasi, L. High-pressure behavior of metylammonium lead iodide (MAPbI3) hybrid perovskite. J. Appl. Phys. 2016, 119, No. 185901.

(18) Jiang, S.; Fang, Y.; Li, R.; Xiao, H.; Crowley, J.; Wang, C.; White, T. J.; Goddard, W. A., III; Wang, Z.; Baikie, T.; et al. Pressure-dependent polymorphism and band-gap tuning of methylammonium lead iodide perovskite. Angew. Chem., Int. Ed. 2016, 55, 6540-6544.

(19) Ou, T.; Yan, J.; Xiao, C.; Shen, W.; Liu, C.; Liu, X.; Han, Y.; Ma, Y.; Gao, C. Visible light 
response, electrical transport, and amorphization in compressed organo lead iodine perovskites. Nanoscale 2016, 8, 11426-11431.

(20) Wang, L.; Wang, K.; Xiao, G.; Zeng, Q.; Zou, B. Pressure-induced structural evolution and band gap shifts of organometal halide perovskite-based methylammonium lead chloride. J. Phys. Chem. Lett. 2016, 7, 5273-5279.

(21) Wang, L.; Wang, K.; Zou, B. Pressure-induced structural and optical properties of organometal halide perovskite-based formamidi-nium lead bromide. J. Phys. Chem. Lett. 2016, 7, $2556-2562$.

(22) Brivio, F.; Frost, J. M.; Skelton, J. M.; Jackson, A. J.; Weber, O. J.; Weller, M. T.; Goñi, A. R.; Leguy, A. M. A.; Barnes, P. R. F.; Walsh, A. Lattice dynamics and vibrational spectra of the orthorhombic, tetragonal, and cubic phases of methylammonium lead iodide. Phys. Rev. B 2015, 92, No. 144308.

(23) Leguy, A. M. A.; Goñi, A. R.; Frost, J. M.; Skelton, J.; Brivio, F.; Rodríguez-Martínez, X.; Weber, O. J.; Pallipurath, A.; Alonso, M. I.; Campoy-Quiles, M.; et al. Dynamic disorder, phonon lifetimes, and the assignment of modes to the vibrational spectra of methylammo-nium lead halide perovskites. Phys. Chem. Chem. Phys. 2016, 18, 27051-27066.

(24) Onoda-Yamamuro, N.; Matsuo, T.; Suga, H. Calorimetric and IR spectroscopic studies of phase transitions in methylammonium trihalogenoplumbates. J. Phys. Chem. Solids 1990, 51, $1383-1395$.

(25) Weller, M. T.; Weber, O. J.; Henry, P. F.; Di Pumpo, A. M.; Hansen, T. C. Complete structure and cation orientation in the perovskite photovoltaic methylammonium lead iodide between 100 and $352 \mathrm{~K}$. Chem. Commun. 2015, 51, 4180-4183.

(26) Leguy, A. M. A.; Frost, J. M.; McMahon, A. P.; Sakai, V. G.; Kochelmann, W.; Law, C.-H.; Li, X.; Foglia, F.; Walsh, A.; O’Regan,

B. C.; et al. The dynamics of methylammonium ions in hybrid organic-inorganic perovskite solar cells. Nat. Commun. 2015, 6, No. 7124.

(27) Bakulin, A. A.; Selig, O.; Bakker, H. J.; Rezus, Y. L. A.; Muller, C.; Glaser, T.; Lovrincic, R.; Sun, Z.; Chen, Z.; Walsh, A.; et al. Real-time observation of organic cation reorientation in methylammonium lead iodide perovskites. J. Phys. Chem. Lett. 2015, 6, 3663-3669.

(28) Pisoni, A.; Jacimovic, J.; Barisic, O. S.; Spina, M.; Gaal, R.; Forro, L.; Horvath, E. Ultra-low thermal conductivity in organic-inorganic hybrid perovskite CH3NH3PbI3. J. Phys. Chem. Lett. 2014, 5, 2488-2492.

(29) Chen, Y.-X.; Ge, Q.-Q.; Shi, Y.; Liu, J.; Xue, D.-J.; Ma, J.-Y.; Ding, J.; Yan, H.-J.; Hu, J.-S.; Wan, L.-J. General space-confined on-substrate fabrication of thickness-adjustable hybrid perovskite single-crystalline thin films. J. Am. Chem. Soc. 2016, 138, 16196-16199.

(30) Angel, R. J.; Bujak, M.; Zhao, J.; Gatta, G. D.; Jacobsen, S. D. Effective hydrostatic limits of pressure media for high-pressure crystallographic studies. J. Appl. Crystallogr. 2007, 40,26-32.

(31) Mao, H.-K.; Xu, J.; Bell, P. M. Calibration of the ruby pressure gauge to $800 \mathrm{kbar}$ under quasi-hydrostatic conditions. J. Geophys. Res. 1986, 91, 4673-4676.

(32) Francisco-López, A.; Charles, B.; Weber, O. J.; Alonso, M. I.; Garriga, M.; Campoy-Quiles, M.; Weller, M. T.; Goñi, A. R. On the Structure-Optical-Properties Relationship of Organic-Cation Solid Solutions of Lead Iodide Perovskites as a Function of Temperature, unpublished work, 2018.

(33) Saba, M.; Cadelano, M.; Marongiu, D.; Chen, F.; Sarritzu, V.; Sestu, N.; Figus, C.; Aresti, M.; Piras, R.; Geddo Lehmann, A.; et al. Correlated electron-hole plasma in organometal perovskites. Nat. Commun. 2014, 5, No. 5049.

(34) Even, J.; Pedesseau, L.; Katan, C. Analysis of multivalley and multibandgap absorption and enhancement of free carriers related to exciton screening in hybrid perovskites. J. Phys. Chem. C 2014, 118, 11566-11572.

(35) Frost, J. M.; Butler, K. T.; Brivio, F.; Hendon, C. H.; van Schilgaarde, M.; Walsh, A. Atomistic origins of high-performance in hybrid halide perovskite solar cells. Nano Lett. 2014, 14, 2584-2590.

(36) Miyata, A.; Mitioglu, A.; Plochocka, P.; Portugall, O.; Wang, J. T.-W.; Stranks, S. D.; Snaith, H. J.; Nicholas, R. J. Direct measurement of the exciton binding energy and effective masses for charge carriers in organic-inorganic tri-halide perovskites. Nat. Phys. 2015, 11, 582-587.

(37) Fang, H.-H.; Raissa, R.; Abdu-Aguye, M.; Adjokatse, S.; Blake,

G. R.; Even, J.; Loi, M. A. Photophysics of organic-inorganic hybrid lead iodide perovskite single 
crystals. Adv. Funct. Mater. 2015, 25, 2378-2385.

(38) Shi, D.; Adinolfi, V.; Comin, R.; Yuan, M.; Alarousu, E.; Buin, A.; Chen, Y.; Hoogland, S.; Rothenberger, A.; Katsiev, K.; et al. Low trap-state density and long carrier diffusion in organolead trihalide perovskite single crystals. Science 2015, 347, 519-522.

(39) Patel, J. B.; Lin, Q.; Zadvorna, O.; Davies, C. L.; Herz, L. M.; Johnston, M. B. Photocurrent spectroscopy of perovskite solar cells over a wide temperature range from 15 to 350 K. J. Phys. Chem. Lett. 2018, 9, 263-268.

(40) Jha, A.; Duan, H.-G.; Tiwari, V.; Nayak, P. K.; Snaith, H. J.; Thorwart, M.; Miller, R. J. D. Direct observation of ultrafast exciton dissociation in lead-iodide perovskite by $2 \mathrm{D}$ electronic spectroscopy. ACS Photonics 2018, 5, 852-860.

(41) D’Innocenzo, V.; Grancini, G.; Alcocer, M. J.-P.; Kandada, A. R. S.; Stranks, S. D.; Lee, M. M.; Lanzani, G.; Snaith, H. J.; Petrozza, A. Excitons versus free charges in organo-lead tri-halide perovskites. Nat. Commun. 2014, 5, No. 3486.

(42) Wu, K.; Bera, A.; Ma, C.; Du, Y.; Yang, Y.; Li, L.; Wu, T. Temperature-dependent excitonic photoluminescence of hybrid organometal halide perovskite films. Phys. Chem. Chem. Phys. 2014, $16,22476-22481$.

(43) Dong, Q.; Fang, Y.; Shao, Y.; Mulligan, P.; Qiu, J.; Cao, L.; Huang, J. Electron-hole diffusion lengths $>175 \mu \mathrm{m}$ in solution-grown CH3NH3PbI3 single crystals. Science 2015, 347, 967-970.

(44) Galkowski, K.; Mitioglu, A. A.; Surrente, A.; Yang, Z.; Maude, D. K.; Kossaki, P.; Eperon, G. E.; Wang, J. T.-W.; Snaith, H. J.; Plochocka, P.; et al. Spatially resolved studies of the phases and morphology of methylammonium and formamidinium lead tri-halide perovskites. Nanoscale 2017, $9,3222-3230$.

(45) Wu, X.; Trinh, M. T.; Niesner, D.; Zhu, H.; Norman, Z.; Owen, J. S.; Yaffe, O.; Kudisch, B. J.; Zhu, X.-Y. Trap states in lead iodide perovskites. J. Am. Chem. Soc. 2015, 137, 2089-2096.

(46) Goñi, A. R.; Syassen, K. Chapter 4 Optical Properties of Semiconductors under Pressure. Semicond. Semimetals 1998, 54, 247- 425 and references therein.

(47) Klingshirn, C. F. Semiconductor Optics; Springer: Berlin, 1997.

(48) We note that the sharp increase in PL intensity before the second phase transition as well as the abrupt intensity reduction in the third high pressure phase is systematically observed for all four measurement series. Although the reason for this behavior is elusive to us, we speculate that it could be due to pressure-induced changes in absorption at the excitation wavelength.

(49) Giorgi, G.; Fujisawa, J.-I.; Segawa, H.; Yamashita, K. Small photocurrent effective masses featuring ambipolar transport in methylammonium lead iodide perovskite: A density functional analysis. J. Phys. Chem. Lett. 2013, 4, 4213-4216.

(50) Even, J.; Pedesseau, L.; Katan, C.; Kepenekian, M.; Lauret, J.-S.; Sapori, D.; Deleporte, E. Solid-state physics perspective on hybrid perovskite semiconductors. J. Phys. Chem. C 2015, 119, $10161-10177$.

(51) Yaffe, O.; Guo, Y.; Tan, L. Z.; Egger, D. A.; Hull, T.; Stoumpos,

C. $\quad$ C.; Zheng, F.; Heinz, T. F.; Kronik, L.; Kanatzidis, M. G.; et al. Local polar fluctuations in lead halide perovskite crystals. Phys. Rev. Lett. 2017, 118, No. 136001.

(52) Sendner, M.; Nayak, P. K.; Egger, D. A.; Beck, S.; Müller, C.; Epding, B.; Kowalsky, W.; Kronik, L.; Snaith, H. J.; Puccia, A.; et al. Optical phonons in methylammonium lead halide perovskites and implications for charge transport. Mater. Horiz. 2016, 3, 613-620.

(53) Goñi, A. R.; Zhou, T.; Schwarz, U.; Kremer, R. K.; Syassen, K. Pressure-temperature phase diagram of the spin-Peierls compound CuGeO3. Phys. Rev. Lett. 1996, 77, 1079-1082.

(54) Yu, P. Y.; Cardona, M. Fundamentals of Semiconductors; Springer: Berlin, 1995.

(55) Charles, B.; Dillon, J.; Weber, O. J.; Islam, M. S.; Weller, M. T. Understanding the stability of mixed A-cation lead iodide perovskites.

J. Mater. Chem. A 2017, 5, 22495-22499.

(56) Jayaraman, A.; Maines, R. G.; Chattopadhyay, T. Pressure- induced structural transitions in PbI2: A high-pressure Raman and optical absorption study. Pramana 1986, 27, 449-457.

(57) Yang, L.; Zhang, Y.; Wang, J.; Wang, Y.; Yu, W. W. Pressure-induced phase transitions of lead iodide. RSC Adv. 2016, 6, 84604- 84609.

(58) Flores-Livas, J. A.; Tomerini, D.; Amsler, M.; Boziki, A.; Rothlisberger, U.; Goedecker, S. Emergence of Hidden Phases of Methylammonium Lead-Iodide (CH3NH3PbI3) upon 
Compression. 2018, arXiv:1806.05858v1 [cond-mat.mtrl-sci]. arXiv.org e-Print archive. https://arxiv.org/abs/1806.05858 (accessed June 15, 2018). 